\title{
Hesitant but vaccinated: assessing COVID-19 vaccine hesitancy among the recently vaccinated
}

\author{
Don E. Willis ${ }^{1} \cdot$ James P. Selig $^{2} \cdot$ Jennifer A. Andersen $^{1} \cdot$ Spencer Hall $^{3}$ • \\ Emily Hallgren $^{1} \cdot$ Mark Williams $^{2} \cdot$ Keneshia Bryant-Moore $^{2} \cdot$ Pearl A. McElfish $^{1} @$
}

Received: 27 August 2021 / Accepted: 4 December 2021 / Published online: 15 January 2022

(C) The Author(s), under exclusive licence to Springer Science+Business Media, LLC, part of Springer Nature 2022

\begin{abstract}
We administered a survey during the fifteenminute wait time after the COVID-19 vaccine was given $(\mathrm{N}=1475)$ to examine attitudes towards COVID-19 vaccines among adults who were vaccinated in Arkansas between April 22nd and July 6th, 2021. We found 60\% of those who had just been vaccinated reported some level of hesitancy, including $10 \%$ who reported being "very hesitant." Hesitancy was not evenly distributed across sociodemographic groups (age, sex, race/ethnicity, and education) and was associated with whether a non-English language is spoken in the home, health care coverage, and flu vaccination over the past five years in bivariate analysis. Generalized ordered logistic regression results reveal associations between the log-ordered odds of COVID-19 vaccine hesitancy and age, sex, race/ethnicity, health care coverage, health literacy, and flu vaccination over the past five years. Surprisingly, a prior COVID-19 diagnosis was not significantly associated with COVID-19 vaccine hesitancy. These results can inform health care and communication strategies. Further attention to "hesitant adopters" can provide insights into the process of overcoming vaccine hesitancy that are critical to vaccine uptake.
\end{abstract}

Keywords COVID-19 - Vaccine hesitancy · Vaccination

Pearl A. McElfish

pamcelfish@uams.edu

1 College of Medicine, University of Arkansas for Medical Sciences Northwest, Fayetteville, AR, USA

2 Fay W. Boozman College of Public Health, University of Arkansas for Medical Sciences, Little Rock, AR, USA

3 Office of Community Health and Research, University of Arkansas for Medical Sciences Northwest, Fayetteville, AR, USA

\section{Introduction}

Vaccine hesitancy was designated as one of the top ten threats to global health by the World Health Organization (WHO) in 2019 (WHO, 2019a). The urgency of understanding and addressing vaccine hesitancy has been amplified by the COVID-19 pandemic which has led to the largest overall decline in United States (US) life expectancy in more than seven decades (Arias et al., 2021). Minority populations have reported greater hesitancy to get the COVID-19 vaccine, with some communities of color being half as likely to be vaccinated as Whites (Khubchandani et al., 2021; Malik et al., 2020; Nguyen et al., 2021; Niño et al., 2021; Willis et al., 2021), but these gaps have narrowed over time (Daly \& Robinson, 2021; Hamel et al., 2021). Vaccine attitudes are often perceived as being on a continuum ranging from complete refusal to active demand (Dubé et al., 2013). However, research defining hesitancy as a behavior, or both an attitude and behavior, faces the problem of conflating issues such as access with the attitude of hesitancy (Quinn et al., 2019). If someone reports being unvaccinated, it may or may not actually reflect their level of vaccine hesitancy (Dubé et al., 2013).

Emerging evidence suggests those who express hesitancy, or an intention to not receive the COVID-19 vaccine, may still get vaccinated. For example, one study shows nearly a quarter of individuals interviewed in January 2021 who reported they would definitely not get the COVID-19 vaccine or would only get it if required have now received at least one dose (Kirzinger et al., 2021). Yet, little is known regarding hesitancy among those who choose to be vaccinated. Two qualitative studies conducted prior to the emergence of COVID-19 focused on hesitancy among parents who accepted a vaccine for their children (Enkel et al., 2018; Walker et al., 2020). One study focused on Australian 
parents making decisions about flu and measles-mumpsrubella vaccinations for their children (Enkel et al., 2018), and the other focused on human papillomavirus vaccination for young adult children (Walker et al., 2020). Both studies demonstrate that hesitancy may co-occur with adherence (Enkel et al., 2018; Walker et al., 2020). However, many questions remain about "hesitant adopters."

Most research on vaccine hesitancy has overlooked those who have been vaccinated, in part because of inconsistency in the operationalization of vaccine hesitancy (Dubé et al., 2013). A review as recent as 2015 found there was no established definition of vaccine hesitancy (MacDonald, 2015). Consistent with the Increasing Vaccination Model (Brewer et al., 2017; WHO, 2019b; WHO EURO, 2011), we view vaccine hesitancy as an attitude or motivational state (Brewer et al., 2017), whereas vaccination is a behavior which may or may not correspond with reported attitudes towards vaccination. Therefore, the behavior of being vaccinated is distinct but not mutually exclusive from the attitude of vaccine hesitancy (Dubé et al., 2013). Further examination of "hesitant adopters"- individuals who are hesitant but vaccinated-can provide insights into the process of overcoming hesitancy to receive a vaccination, which is critical to vaccine uptake.

Rather than assuming those who receive a COVID-19 vaccine are not hesitant, we asked the following questions: (1) How prevalent is hesitancy towards the COVID-19 vaccine among those who have recently been vaccinated? (2) How does this COVID-19 vaccine hesitancy among the recently vaccinated vary across sociodemographic groups? (3) How does this COVID-19 vaccine hesitancy among the recently vaccinated relate to other factors such as prior diagnosis of COVID-19, health care coverage, health literacy, and flu vaccination behavior over the past five years?

We examined these questions in the context of a state which has COVID-19 vaccination rates substantially lower than the national average. As of August 24th 2021, only $39 \%$ of the total population of Arkansas was fully vaccinated, compared to $52 \%$ of the US population (HHS \& CDC, 2021). States with low vaccination rates have experienced a disproportionate burden of COVID-19 hospitalizations and deaths, especially since the emergence of the Delta variant (Leatherby \& Walker, 2021a, b). Thus, understanding COVID-19 vaccine hesitancy in states such as Arkansas is particularly pressing.

\section{Methods}

Respondents were recruited at more than 30 vaccination sites across the state of Arkansas where the COVID-19 vaccine was given. Vaccination sites include drive-through clinic locations and vaccination events in the community and at faith-based organizations. Respondents had to be 18 years of age or older, be recently vaccinated, and speak or read English, Spanish, or Marshallese to be included in the study.

The consent and survey were administered during the fifteen-minute wait time after the COVID-19 vaccine was given using REDCap (Harris et al., 2009). Participants were seated after receiving their vaccination, provided information about the survey, and invited to participate. The study included an incentive of entry into a raffle for a $\$ 100$ Walmart gift card. The consent information and survey were available in English, Spanish, and Marshallese. Respondents could document consent and complete the survey on their smartphone by scanning a QR code or utilizing a sterilized iPad provided by study staff. Respondents could also complete the study with assistance of a bilingual staff member asking them the questions verbally. Respondents had the ability to refuse (as indicated by skipping or selecting "prefer not to answer") questions. Data were collected between April 22nd and July 6th, 2021. The study was approved by the University of Arkansas for Medical Sciences Institutional Review Board (IRB \#262645).

\section{Measures}

The survey included sociodemographic questions, as well as questions to assess COVID-19 vaccine hesitancy, prior COVID-19 diagnosis, health care coverage, health literacy, and past history of flu vaccinations.

\section{COVID-19 vaccine hesitancy}

To measure the dependent variable of COVID-19 vaccine hesitancy, we modified an existing single-item measure of vaccine hesitancy to specifically capture attitudes about the COVID-19 vaccine. The original question asked, "Overall, how hesitant are you about getting vaccinations?" (Quinn et al., 2017, 2019). We modified this to ask respondents to think specifically about how hesitant they were to receive the COVID-19 vaccine. Respondents were asked, "Thinking specifically about the COVID-19 vaccine, how hesitant were you about getting vaccinated?" Response options included the following: "not at all hesitant," "a little hesitant," "somewhat hesitant," and "very hesitant."

\section{Sociodemographics}

Sociodemographic information, including age, sex, race, education, marital status, and whether non-English languages were spoken in the home, was collected. Sociodemographic information other than age was assessed using standard questions from the Behavioral Risk Factor Survey (CDC, 2020). Age was measured using a question from the General Social Survey as a continuous variable calculated from reported date of birth or respondents' best 
estimates of their age (Smith et al., 2019). Sex was measured as a categorical variable of either female or male with the option to self-describe-responses from nonbinary respondents were too few to include in the analysis. Respondents were asked to identify their race and could select all that apply from a list of valid responses: American Indian or Alaska Native (AIAN), Asian, Black/ African American, Native Hawaiian or Pacific Islander (NHPI), White, or Other. These responses were combined with a response to a question about ethnicity to indicate whether each individual was non-Hispanic Black/African American, non-Hispanic NHPI, non-Hispanic White, nonHispanic Multiracial or other racial group, or Hispanic or Latino. Responses from AIAN, Asian, and multiracial respondents were too few to keep these categories separate from others in analyses, so they were combined. Although combining these groups is problematic, we decided this option was preferable to excluding them from the analysis. To assess educational attainment, respondents selected the closest fit from an ordinal set of responses. Possible responses included "never attended or only kindergarten," "grades 1 through 8," "grades 9 through 11," "grade 12 or GED," "some college or technical school," or "college 4 years or more." There were few respondents who selected options below "less than a high school," and these responses were combined into a single "less than high school" category. Respondents were presented a question about relationship status. For analysis, responses were coded into married and non-married respondents. Nonmarried respondents include any respondent who provided a valid response other than "married." Respondents were asked if they spoke a language other than English at home, with possible responses of "yes" and "no" (American Community Survey, 2019).

\section{Prior COVID-19 diagnosis}

To assess whether individuals had been diagnosed with COVID-19 prior to their vaccination, respondents were asked, "Has a doctor or another health care professional diagnosed you with the coronavirus (COVID-19)?" Possible responses included "yes" or "no."

\section{Health care coverage}

To assess health care coverage, we used a standard measure from the Behavioral Risk Factor Surveillance Survey (CDC, 2020). Respondents were asked, "Do you have any kind of health care coverage, including health insurance, prepaid plans such as HMOs, or government plans such as
Medicare, or Indian Health Service?" Possible responses included "yes" or "no."

\section{Health literacy}

A single-item screener of health literacy was used. Health literacy screeners have been validated among low-income and Spanish-speaking patients (Chew et al., 2008; Hadden et al., 2019; Ylitalo et al., 2018), and evidence suggests that a single item is effective at detecting limited to marginal health literacy with reasonable specificity (Wallace et al., 2006). This question asked respondents, "How confident are you filling out medical forms by yourself?" Possible responses included "extremely," "quite a bit," "somewhat," "a little bit," or "not at all."

\section{Flu vaccine behavior}

An existing measure of flu vaccination behavior was used to provide insight into whether individuals typically received a flu shot over the past five years (Quinn et al., 2017). The question asked respondents, "How often in the past 5 years have you gotten a seasonal flu vaccine?" Possible responses included "every year," "most years but not all," "once or twice," and "never."

\section{Statistical analyses}

We present descriptive statistics for all variables in the study. Spearman correlations and Kruskal Wallis H tests were performed to assess bivariate relationships with COVID-19 vaccine hesitancy. Due to the ordinal nature of the dependent variable, a generalized ordered logistic regression with partial proportional odds (Williams, 2006) was used to analyze multivariable relationships with COVID-19 vaccine hesitancy. We made the decision to use a partial proportional odds model following a Brant test which revealed that age, health care coverage, and health literacy violated the proportional odds assumption in an ordered logistic regression. The generalized ordered logistic regression allowed us to relax the proportional odds assumption for age, health care coverage, and health literacy, while maintaining it for other variables (Williams, 2006). All tests were two-tailed, with a standard significance level of $p<0.05$. Statistical analysis was performed on Stata SE/15.1.

\section{Results}

Our analytical sample includes 1475 respondents. Among these respondents, $91 \%$ answered every item in the survey. In Table 1, we present the descriptive statistics of this sample $(\mathrm{N}=1475)$. The average respondent was 38 years old, 
Table 1 Descriptives of recently vaccinated Arkansans

\begin{tabular}{|c|c|c|c|c|}
\hline & Frequency & $\%$ or $\bar{x}$ & S.D & Range \\
\hline Age & 1475 & 38.18 & 13.44 & $18-94$ \\
\hline Sex & 1463 & & & $0-1$ \\
\hline Female & 838 & 57 & & \\
\hline Male & 625 & 43 & & \\
\hline Race/Ethnicity & 1457 & & & $1-5$ \\
\hline NH Black/African American & 72 & 5 & & \\
\hline NH Native Hawaiian or Pacific Islander & 170 & 12 & & \\
\hline NH White & 612 & 42 & & \\
\hline Hispanic/Latinx & 496 & 34 & & \\
\hline NH Multiracial and Other Racial Groups & 107 & 7 & & \\
\hline Education & 1428 & & & $1-4$ \\
\hline Less than high school & 227 & 16 & & \\
\hline High school & 359 & 25 & & \\
\hline Some college & 338 & 24 & & \\
\hline Four-year degree & 504 & 35 & & \\
\hline Marital Status & 1432 & & & $0-1$ \\
\hline Married & 709 & 50 & & \\
\hline Not married & 723 & 50 & & \\
\hline Non-English Language in Home & 1465 & & & $0-1$ \\
\hline Yes & 605 & 41 & & \\
\hline No & 860 & 59 & & \\
\hline Prior COVID-19 Diagnosis & 1452 & & & $0-1$ \\
\hline Yes & 300 & 21 & & \\
\hline No & 1152 & 79 & & \\
\hline Health Care Coverage & 1379 & & & $0-1$ \\
\hline Yes & 969 & 70 & & \\
\hline No & 410 & 30 & & \\
\hline Health Literacy (medical forms) & 1441 & & & $0-4$ \\
\hline Not at all & 30 & 2 & & \\
\hline A little bit & 59 & 4 & & \\
\hline Somewhat & 125 & 9 & & \\
\hline Quite a bit & 337 & 23 & & \\
\hline Extremely & 890 & 62 & & \\
\hline Flu Vaccination (past 5 years) & 1439 & & & $0-3$ \\
\hline Never & 366 & 25 & & \\
\hline Once or twice & 338 & 24 & & \\
\hline Most years but not all & 314 & 22 & & \\
\hline Every year & 421 & 29 & & \\
\hline COVID-19 Vaccine Hesitancy & 1459 & & & $0-3$ \\
\hline Not at all hesitant & 587 & 40 & & \\
\hline A little hesitant & 451 & 31 & & \\
\hline Somewhat hesitant & 271 & 19 & & \\
\hline Very hesitant & 150 & 10 & & \\
\hline
\end{tabular}

$\mathrm{NH}=$ non-Hispanic

and approximately half were female (57\%). The sample was racially and linguistically diverse with less than half of the population identifying as White (42\%) and slightly over half speaking only English in the home (59\%). Twenty-nine percent received their flu vaccinations every year over the past five years. Approximately one in five (21\%) reported they had been diagnosed with COVID-19. Although $40 \%$ reported themselves to be "not at all hesitant" about getting 
the COVID-19 vaccine, the remaining $60 \%$ reported some level of hesitancy: $31 \%$ were "a little hesitant," $19 \%$ were "somewhat hesitant," and 10\% were "very hesitant."

In Table 2, we present the bivariate analysis between COVID-19 vaccine hesitancy and all independent variables. Spearman correlation analysis demonstrated age was negatively associated with COVID-19 vaccine hesitancy $(\mathrm{rs}(1452)=-0.064 ; p=0.015)$. Kruskal Wallis $\mathrm{H}$ tests demonstrated there was a difference in vaccine hesitancy by $\operatorname{sex}\left(\chi^{2}(1)=5.94 ; p<0.015\right)$, race $\left(\chi^{2}(4)=37.44 ; p<0.001\right)$, education $\left(\chi^{2}(3)=10.83\right.$; $p=0.013)$, whether a language other than English was

Table 2 Bivariate relationships with hesitancy among the recently vaccinated

\begin{tabular}{|c|c|c|c|c|c|}
\hline & $\begin{array}{l}\text { Not at all } \\
\text { Freq(row\%) }\end{array}$ & A little Freq(row\%) & $\begin{array}{l}\text { Somewhat } \\
\text { Freq(row\%) }\end{array}$ & Very Freq(row\%) & $p$-value \\
\hline${ }^{\text {a Age (mean) }}$ & 40 & 37 & 37 & 39 & .015 \\
\hline Sex & & & & & .015 \\
\hline Female & $302(37)$ & $273(33)$ & 157(19) & $91(11)$ & \\
\hline Male & $275(44)$ & $174(28)$ & $114(18)$ & $58(9)$ & \\
\hline Race/Ethnicity & & & & & .000 \\
\hline NH Black/African American & $17(24)$ & $16(22)$ & $19(26)$ & $20(28)$ & \\
\hline NH Native Hawaiian or Pacific Islander & $94(57)$ & $35(21)$ & $27(16)$ & $10(6)$ & \\
\hline NH White & $230(38)$ & $199(33)$ & $116(19)$ & $64(11)$ & \\
\hline Hispanic/Latinx & 202(42) & $160(33)$ & $77(16)$ & $47(10)$ & \\
\hline NH Multiracial and Other Racial Groups & $39(37)$ & $37(35)$ & $24(23)$ & $5(5)$ & \\
\hline Education & & & & & .013 \\
\hline Less than high school & 111(49) & $67(30)$ & $28(12)$ & $19(8)$ & \\
\hline High school & 139(39) & $110(31)$ & $66(19)$ & $37(11)$ & \\
\hline Some college & $135(40)$ & $96(29)$ & $64(19)$ & $40(12)$ & \\
\hline Four-year degree & 184(37) & $167(33)$ & $103(21)$ & $48(10)$ & \\
\hline Marital Status & & & & & .105 \\
\hline Married & $297(43)$ & $213(31)$ & $120(17)$ & $67(10)$ & \\
\hline Not married & $276(38)$ & $229(32)$ & 139(19) & $75(10)$ & \\
\hline Prior COVID-19 Diagnosis & & & & & .915 \\
\hline Yes & 121(40) & $90(30)$ & $56(19)$ & $32(11)$ & \\
\hline No & $457(40)$ & $354(31)$ & 209(18) & $116(10)$ & \\
\hline Non-English Language in Home & & & & & .001 \\
\hline Yes & $266(45)$ & 177(30) & $106(18)$ & $45(8)$ & \\
\hline No & $317(37)$ & $270(32)$ & $162(19)$ & $103(12)$ & \\
\hline Health Care Coverage & & & & & .016 \\
\hline Yes & $366(38)$ & $309(32)$ & $190(20)$ & $96(10)$ & \\
\hline No & $190(47)$ & $109(27)$ & $59(15)$ & $46(11)$ & \\
\hline Health Literacy (medical forms) & & & & & .122 \\
\hline Not at all & $8(27)$ & $16(53)$ & $2(7)$ & $4(13)$ & \\
\hline A little bit & $24(41)$ & $20(34)$ & $7(12)$ & $8(14)$ & \\
\hline Somewhat & $41(33)$ & $36(29)$ & $35(28)$ & $13(10)$ & \\
\hline Quite a bit & $136(41)$ & $119(36)$ & $57(17)$ & $19(6)$ & \\
\hline Extremely & $363(41)$ & $251(28)$ & 167(19) & 101(12) & \\
\hline Flu Vaccination (past 5 years) & & & & & .000 \\
\hline Never & 140(39) & $90(25)$ & $76(21)$ & $55(15)$ & \\
\hline Once or twice & 111(33) & $115(35)$ & $69(21)$ & $38(11)$ & \\
\hline Most years but not all & $116(37)$ & $116(37)$ & $58(15)$ & $31(7)$ & \\
\hline Every year & $211(50)$ & $116(28)$ & $61(15)$ & $31(7)$ & \\
\hline
\end{tabular}

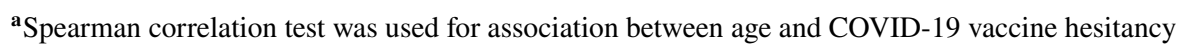

Percentages may not sum to 100 due to rounding

$\mathrm{NH}=$ non-Hispanic 
spoken in the home $\left(\chi^{2}(1)=10.56 ; p=0.001\right)$, health care coverage $\left(\chi^{2}(1)=5.82 ; p=0.016\right)$, and flu vaccination behavior over the past five years $\left(\chi^{2}(1)=28.06 ; p<0.001\right)$. Reports of being "not at all" hesitant were most prevalent among men, NHPIs, and those with less than a high school degree, who speak a language other than English in the home, who do not have health care coverage, and who report flu vaccination every year in the past five years. Black/African American respondents had the lowest percentage (24\%) of reporting they were "not at all" hesitant to receive the vaccine and the highest percentage (28\%) of reporting they were very hesitant. Marital status, prior COVID-19 diagnosis, and health literacy were not associated with COVID-19 vaccine hesitancy.

In Table 3, we present the ordered log-odds regression coefficients from a generalized ordered logistic regression using a partial proportional odds model. These coefficients indicate the increase or decrease in log-odds of reporting a higher level of vaccine hesitancy. The ordered log-odds of vaccine hesitancy were associated with age, sex, race, health care coverage, health literacy, and flu vaccination behaviors over the past five years. As age increased, respondents were

Table 3 Generalized ordered logistic regression of hesitancy among recently vaccinated

\begin{tabular}{|c|c|c|c|c|c|}
\hline & Coef & S.E & p-value & $95 \%$ Conf & Interval \\
\hline \multicolumn{6}{|l|}{ age } \\
\hline Not at all hesitant & -.012 & .005 & .011 & -.022 & -.003 \\
\hline A little hesitant & -.000 & -.020 & .982 & -.010 & .010 \\
\hline Somewhat hesitant & .015 & .007 & .043 & .000 & .029 \\
\hline \multicolumn{6}{|l|}{ Sex (Ref: Male) } \\
\hline Female & .377 & .110 & .001 & .162 & .593 \\
\hline \multicolumn{6}{|l|}{ Race/Ethnicity (Ref: White) } \\
\hline NH Black/African American & .956 & .246 & .000 & .475 & 1.437 \\
\hline NH Native Hawaiian or Pacific Islander & -.970 & .274 & .000 & -1.508 & -.432 \\
\hline Hispanic/Latinx & -.200 & .197 & .309 & -.586 & .186 \\
\hline NH Multiracial and Other Racial Groups & -.092 & .222 & .680 & -.527 & .344 \\
\hline \multicolumn{6}{|l|}{ Education (Ref: Four-year degree) } \\
\hline Less than high school & -.186 & .211 & .377 & -.599 & .227 \\
\hline High school & .192 & .155 & .215 & -.111 & .495 \\
\hline Some college & .064 & .142 & 653 & -.214 & .341 \\
\hline \multicolumn{6}{|l|}{ Marital Status (Ref: Not married) } \\
\hline Married & -.009 & .115 & .939 & -.234 & .216 \\
\hline \multicolumn{6}{|l|}{ Prior COVID-19 Diagnosis } \\
\hline Yes & .194 & .135 & .151 & -.071 & .459 \\
\hline \multicolumn{6}{|l|}{ Non-English Language in Home } \\
\hline Yes & -.046 & .165 & .780 & -.369 & .277 \\
\hline \multicolumn{6}{|l|}{${ }^{a}$ Health Care Coverage (Ref: No coverage) } \\
\hline Not at all hesitant & .291 & .145 & .044 & .007 & .574 \\
\hline A little hesitant & .049 & .158 & .757 & -.261 & .359 \\
\hline Somewhat hesitant & -.476 & .216 & .027 & -.899 & -.053 \\
\hline \multicolumn{6}{|l|}{ a'Health Literacy (medical forms) } \\
\hline Not at all hesitant & -.226 & .071 & .002 & -.365 & -.086 \\
\hline A little hesitant & -.063 & .076 & .401 & -.211 & .085 \\
\hline Somewhat hesitant & -.013 & .110 & .908 & -.228 & .202 \\
\hline \multicolumn{6}{|l|}{ Flu Vaccination (past 5 years) } \\
\hline Flu vaccination over past 5 years & -.226 & .048 & .000 & -.320 & -.132 \\
\hline Constant (Not at all hesitant) & 1.637 & .361 & .000 & .930 & 2.344 \\
\hline Constant (A little hesitant) & -.603 & .373 & .105 & -1.334 & .127 \\
\hline Constant (Somewhat hesitant) & -2.272 & .506 & .000 & -3.263 & -1.281 \\
\hline Pseudo r-squared & .038 & Number of obs & & 1237 & \\
\hline Chi-square & 118.98 & Prob $>$ chi 2 & & .000 & \\
\hline
\end{tabular}

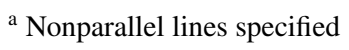

$\mathrm{NH}=$ non-Hispanic 
less likely to report some level of hesitancy compared to no hesitancy ( $\beta=-0.012 ; 95 \%$ CI $[-0.022,-0.003])$; however, as age increased, people were more likely to report being very hesitant rather than somewhat hesitant $(\beta=0.015 ; 95 \%$ CI $[0.000,0.029])$. Women were more likely to report higher levels of vaccine hesitancy compared to men $(\beta=0.377$; 95\% CI $[0.162,0.593])$. Black/African American respondents were more likely to report higher levels of hesitancy ( $\beta=0.956$; 95\% CI $[0.475,1.437])$, and NHPI respondents were less likely to report a higher level of hesitancy ( $\beta=-0.970 ; 95 \%$ CI $[-1.508,-0.432])$ compared to White respondents.

Respondents who had health care coverage were more likely to report some level of hesitancy $(\beta=0.291 ; 95 \% \mathrm{CI}$ $[0.007,0.574])$; however, respondents with health care coverage were less likely to report being very hesitant compared to somewhat hesitant $(\beta=-0.476 ; 95 \%$ CI $[-0.899$, $-0.053])$. Health literacy was associated with vaccine hesitancy; as health literacy increased, respondents were less likely to report some level of hesitancy ( $\beta=-0.226$; $95 \%$ CI $[-0.365,-0.086])$. As the number of years respondents reported receiving a flu vaccination increased, the less likely they were to report higher levels of hesitancy $(\beta=-0.226$; 95\% CI [-0.320, -0.132]). Education, marital status, prior COVID-19 diagnosis, and speaking a non-English language in the home were not significantly associated with COVID19 vaccine hesitancy.

\section{Discussion}

We found hesitancy towards the COVID-19 vaccine was prevalent among recently vaccinated Arkansans. Surprisingly, $60 \%$ of recently vaccinated individuals showed some level of hesitancy towards the COVID-19 vaccination. Among these "hesitant adopters," the majority were "a little hesitant" (31\%) or "somewhat hesitant" (19\%); however, $10 \%$ said they were "very hesitant." The co-occurrence of vaccine hesitancy and the behavior of vaccination suggests that hesitant individuals can overcome their hesitancy enough to receive the vaccine.

Hesitancy varied by race/ethnicity. Black/African American respondents were significantly more hesitant than White respondents or any other racial/ethnic group, with $76 \%$ of Black/African American respondents who had recently received a COVID-19 vaccination reporting some level of hesitancy and 28\% reporting they were "very hesitant" even after getting the COVID-19 vaccination. This finding is encouraging given prior studies have documented that Black/African American individuals report significantly more hesitancy compared to other racial/ethnic groups (Latkin et al., 2021; Malik et al., 2020; Willis et al., 2021). Historical and ongoing racism has undermined the trustworthiness of medical institutions (Warren et al., 2020; H. A. Washington, 2006; J. Washington, 2020), which continues to feed into vaccine hesitancy among Black/African American individuals (Savoia et al., 2021). However, past studies have conflated the attitude of hesitancy with the behaviors of receiving or not receiving a vaccine (Quinn et al., 2019). It is important to continue to address vaccine hesitancy; however, our study suggests many Black/African American respondents may choose to get vaccinated despite feeling hesitant.

Interestingly, NHPI respondents reported less hesitancy compared to White respondents. This could be because a large portion of NHPI individuals who are hesitant are not getting vaccinated, or it could be due to intensive, culturally appropriate COVID-19 vaccine outreach and education efforts implemented in Arkansas, which may have reduced hesitancy (McElfish et al., 2021a, 2021b). What is known about hesitancy among NHPIs has been somewhat inconsistent. For example, research on NHPIs tends to aggregate them with Asian Americans (Chang et al., 2020). Studies combining these groups report they have low levels of vaccine hesitancy (Niño et al., 2021); however, studies which distinguish them reveal NHPIs have much higher COVID-19 vaccine hesitancy compared to Asian Americans (Ta Park et al., 2021).

Although a higher proportion of Hispanic/Latinx respondents reported no hesitancy at all compared to Whites, the association was not significant when adjusting for other factors. Similar to NHPIs, research on vaccine hesitancy among Hispanics/Latinx has been mixed. Some research has documented no significant differences in COVID-19 vaccine hesitancy between Hispanic/Latinx individuals and White individuals (Willis et al., 2021), while other studies found that Hispanic/Latinx individuals report significantly higher vaccine hesitancy (Nguyen et al., 2021) or lower probability of intention to vaccinate (Latkin et al., 2021; Niño et al., 2021).

Females reported greater hesitancy compared to males after having received a COVID-19 vaccine, even after adjusting for other independent variables. This finding is consistent with prior research assessing differences in vaccine hesitancy between men and women in the general population (Latkin et al., 2021). The present study documents there are still differences in hesitancy even among men and women who have decided to be vaccinated. Calarco and Anderson (2021) attribute gendered differences in opposition to new public health initiatives like vaccination to the gendered pressures mothers experience. These gendered pressures result in mothers supporting initiatives they control and perceive to be useful but opposing measures like vaccination which they perceive to be an uncontrollable risk (Calarco \& Anderson, 2021). 
Hesitancy towards the COVID-19 vaccine was associated with whether a non-English language is spoken in the home, health care coverage, and flu vaccination behaviors over the past five years. Although health literacy was not associated with hesitancy in bivariate analysis, it was significant when adjusting for other independent variables - the opposite was true for non-English languages spoken in the home, which was not significant in the regression analysis. Reviews of vaccine hesitancy in general have noted the importance of factors such as access to health care and potential language barriers and health literacy in communicating information about the vaccine (MacDonald, 2015). We were surprised to find having been diagnosed with COVID-19 prior to vaccination was not associated with hesitancy towards receiving the vaccine given some of the misinformation about vaccines has focused on whether those with prior infection should receive the COVID-19 vaccine (Mandavilli, 2020). The majority of research on COVID-19 vaccine hesitancy has not assessed differences across languages spoken in the household, prior COVID-19 diagnosis, or health care coverage, particularly among those who have recently been vaccinated.

These findings have implications for messaging within vaccine clinics and outreach programs. The prevalence of hesitancy among those being vaccinated suggests health care providers should be considerate of hesitation as they answer patient questions. Assuming those who choose to be vaccinated are fully confident in their decision is a lost opportunity to ease any concerns, answer questions, and help encourage them to return for their second dose (if needed). Findings suggest hesitancy towards vaccination should not be conflated with vaccine refusal, just as vaccine compliance should not be conflated with a lack of hesitancy (Bedford et al., 2018). Assuming the unvaccinated are hesitant dismisses issues of access and convenience, while assuming the vaccinated are not hesitant dismisses any ongoing concerns people may have leading up to and after vaccination (Quinn et al., 2019). Vaccine attitudes and behaviors are not static (Enkel et al., 2018), and they do not always relate to one another in ways we might expect (Brewer et al., 2017). These points are foundational for any attempts at shifting vaccine attitudes or behaviors. Further examination of "hesitant adopters" is necessary to provide insights into the process of overcoming vaccine hesitancy which is critical to identifying points of intervention. Although hesitancy often differs from one vaccine to another, investigating this group further may also allow for a better understanding of what it means to become vaccinated despite vaccine hesitancy.

\section{Strengths and limitations}

This study does have limitations. The data are cross-sectional; thus, the results cannot establish causality. Moreover, the sample was not randomly selected and only included vaccination sites in Arkansas and may not be generalizable to all recently vaccinated individuals. We encourage others to investigate this topic in other locations and contexts. Finally, we relied on self-reported measures for variables such as health literacy and prior diagnosis of COVID-19, and our measure of health literacy relied on a single-item. Future research aimed at understanding the effectiveness of vaccination interventions will require longitudinal designs and the inclusion of both vaccinated and unvaccinated individuals. Although we find that individuals may be hesitant even as they are being vaccinated, future research should explore questions related to the presence of hesitancy at multiple time points after vaccination to better understand the durability or fluctuation of these attitudes.

A major strength of this study is it addresses questions related to COVID-19 vaccine hesitancy among the recently vaccinated, a group which has been overlooked in the literature on vaccine hesitancy in part due to inconsistencies in conceptualization and operationalization of hesitancy. This is the first study to examine hesitancy among a sample of recently vaccinated individuals, providing novel insights into the prevalence and variation of hesitancy among hesitant adopters. The study utilized a large and diverse sample. Moreover, the study was conducted in a context where vaccination rates have been lagging behind national averages and where understanding COVID-19 vaccine uptake and hesitancy towards the COVID-19 vaccine is urgent.

Acknowledgments The authors would like to thank those who participated in this study, including both those who received their vaccination and took the survey as well as the health care and community workers whose efforts were critical to conducting this research.

Author contributions All authors contributed to the study conception and design. Material preparation, data collection, and analysis were performed by Don E. Willis, Pearl A. McElfish, James P. Selig, Jennifer A. Andersen, and Spencer Hall. The first draft of the manuscript was written by Don E. Willis, and all authors commented on previous versions of the manuscript. All authors read and approved the final manuscript.

Funding The authors disclosed receipt of the following financial support for the research, authorship, and/or publication of this article: The research described was supported by the Translational Research Institute (TRI) [grant number UL1 TR003107] through the National Center for Advancing Translational Sciences of the National Institutes of Health (NIH) and through an award from NIH Community Engagement Alliance (CEAL) Against COVID-19 Disparities [grant number 1OT2HL161580-01]. The content is solely the responsibility of the authors and does not necessarily represent the official views of the NIH.

\section{Declarations}

Conflict of interest The authors have no conflicts of interest to declare. 
Human and animal participants All procedures followed were in accordance with ethical standards of the responsible committee on human experimentation (institutional and national) and with the Helsinki Declaration of 1975, as revised in 2000.

Informed consent Informed consent was obtained from all individual participants included in the study.

Ethical approval All study procedures were approved by the University of Arkansas for Medical Sciences Institutional Review Board (IRB \#262645).

Consent to participate All relevant ethical regulations have been followed and participants have completed informed consent.

\section{Consent for publication Not applicable.}

Availability of data and material The deidentified data underlying the results presented in this study may be made available upon request from the corresponding author Dr. Pearl A. McElfish, at pamelfish@ uams.edu. The data are not publicly available in accordance with funding requirements and participant privacy.

Code availability Statistical analysis was performed on Stata SE/15.1.

\section{References}

American Community Survey. (2019). U.S. Census Bureau QuickFacts: United States. https://www.census.gov/quickfacts/fact/ table/AR,US/PST045219

Arias, E., Betzaida, T.-V., Ahmad, F., \& Kochanek, K. (2021). Provisional life expectancy estimates for 2020. National Center for Health Statistics (U.S.). https://doi.org/10.15620/cdc:107201

Bedford, H., Attwell, K., Danchin, M., Marshall, H., Corben, P., \& Leask, J. (2018). Vaccine hesitancy, refusal and access barriers: The need for clarity in terminology. Vaccine, 36, 6556-6558. https://doi.org/10.1016/j.vaccine.2017.08.004

Brewer, N. T., Chapman, G. B., Rothman, A. J., Leask, J., \& Kempe, A. (2017). Increasing vaccination: Putting psychological science into action. Psychological Science in the Public Interest: A Journal of the American Psychological Society, 18, 149-207. https://doi.org/ $10.1177 / 1529100618760521$

Calarco, J. M., \& Anderson, E. M. (2021). "I'm Not Gonna Put That On My Kids": Gendered Opposition to New Public Health Initiatives. SocArXiv. https://doi.org/10.31235/osf.io/tv8zw

CDC. (2020). Behavioral Risk Factor Surveillance System Survey Questionnaire. U.S. Department of Health and Human Services, Centers for Disease Control and Prevention.

Chang, C. R., Penaia, C., \& Thomas, K. (2020). Count Native Hawaiian And Pacific Islanders In COVID-19 Data-It's An OMB Mandate I Health Affairs Blog. https://www.healthaffairs.org/ do/https://doi.org/10.1377/hblog20200825.671245/full/

Chew, L. D., Griffin, J. M., Partin, M. R., Noorbaloochi, S., Grill, J. P., Snyder, A., Bradley, K. A., Nugent, S. M., Baines, A. D., \& VanRyn, M. (2008). Validation of screening questions for limited health literacy in a large VA outpatient population. Journal of General Internal Medicine, 23, 561-566. https://doi.org/10.1007/ s11606-008-0520-5

Daly, M., \& Robinson, E. (2021). Willingness to vaccinate against COVID-19 in the U.S.: Representative longitudinal evidence from
April to October 2020. American Journal of Preventive Medicine, 60, 766. https://doi.org/10.1016/j.amepre.2021.01.008

Dubé, E., Laberge, C., Guay, M., Bramadat, P., Roy, R., \& Bettinger, J. A. (2013). Vaccine hesitancy: An overview. Human Vaccines \&amp; Immunotherapeutics, 9, 1763-1773. https://doi.org/10. 4161/hv.24657

Enkel, S. L., Attwell, K., Snelling, T. L., \& Christian, H. E. (2018). 'Hesitant compliers': Qualitative analysis of concerned fullyvaccinating parents. Vaccine, 36, 6459-6463. https://doi.org/10. 1016/j.vaccine.2017.09.088

Hadden, K. B., Prince, L. Y., Rojo, M. O., Selig, J. P., \& McElfish, P. A. (2019). Screening patients who speak spanish for low health literacy. Health Literacy Research and Practice, 3(2), e110-e116. https://doi.org/10.3928/24748307-20190408-03

Hamel, L., Lopes, L., Sparks, G., Kirzinger, A., Kearney, A., Stokes, M., \& Brodie, M. (2021). KFF COVID-19 Vaccine Monitor: October 2021. KFF. https://www.kff.org/coronavirus-covid-19/ poll-finding/kff-covid-19-vaccine-monitor-october-2021/

Harris, P. A., Taylor, R., Thielke, R., Payne, J., Gonzalez, N., \& Conde, J. G. (2009). Research electronic data capture (REDCap)-A metadata-driven methodology and workflow process for providing translational research informatics support. Journal of Biomedical Informatics, 42, 377-381. https:// doi.org/10.1016/j.jbi.2008.08.010

HHS, \& CDC. (2021). COVID-19 State Profile Report-Combined Set. https://healthdata.gov/Community/COVID-19-State-Profi le-Report-Combined-Set/5mth-2h7d

Khubchandani, J., Sharma, S., Price, J. H., Wiblishauser, M. J., Sharma, M., \& Webb, F. J. (2021). COVID-19 vaccination hesitancy in the United States: A rapid national assessment. Journal of Community Health, 46, 270-277. https://doi.org/10. 1007/s10900-020-00958-x

Kirzinger, A., Sparks, G., \& Brodie, M. (2021). KFF COVID-19 Vaccine Monitor: In Their Own Words, Six Months Later. KFF. https://www.kff.org/coronavirus-covid-19/poll-finding/kff-covid19-vaccine-monitor-in-their-own-words-six-months-later/

Latkin, C., Dayton, L., Yi, G., Konstantopoulos, A., \& Boodram, B. (2021). Trust in a COVID-19 vaccine in the U.S.: A social-ecological perspective. Social Science \&amp; Medicine, 270, 113684. https://doi.org/10.1016/j.socscimed.2021.113684

Leatherby, L., \& Walker, A. S. (2021a). Less vaccinated states are feeling the force of Delta's surge in the U.S. The New York Times. https://www.nytimes.com/2021/07/17/world/less-vaccinatedstates-are-feeling-the-force-of-deltas-surge-in-the-us.html

Leatherby, L., \& Walker, A. S. (2021b). Unvaccinated States Feel Brunt of Delta-Led Covid Uptick. The New York Times. https://www. nytimes.com/interactive/2021/07/17/us/delta-variant-us-growth. html

MacDonald, N. E. (2015). Vaccine hesitancy: Definition, scope and determinants. Vaccine, 33, 4161-4164. https://doi.org/10.1016/j. vaccine.2015.04.036

Malik, A. A., McFadden, S. M., Elharake, J., \& Omer, S. B. (2020). Determinants of COVID-19 vaccine acceptance in the US. EClinicalMedicine. https://doi.org/10.1016/j.eclinm.2020.100495

Mandavilli, A. (2020). 'Natural immunity' from Covid is not safer than a vaccine. The New York Times. https://www.nytimes.com/2020/ 12/05/health/covid-natural-immunity.html

McElfish, P. A., Cleek, A. B., Willis, D. E., Purvis, R. S., \& James, L. P. (2021a). Leveraging community engagement capacity to address COVID-19 disparities among Pacific Islander and Latinx Communities in Arkansas. Journal of Clinical and Translational Science, 5(1). https://doi.org/10.1017/cts.2020.562

McElfish, P. A., Purvis, R., Willis, D. E., \& Riklon, S. (2021b). COVID-19 disparities among marshallese pacific islanders. Preventing Chronic Disease, 18, 200407. https://doi.org/10.5888/ pcd18.200407 
Nguyen, L. H., Joshi, A. D., Drew, D. A., Merino, J., Ma, W., Lo, C.-H., Kwon, S., Wang, K., Graham, M. S., Polidori, L., Menni, C., Sudre, C. H., Anyane-Yeboa, A., Astley, C. M., Warner, E. T., Hu, C. Y., Selvachandran, S., Davies, R., Nash, D., Consortium, on behalf of the C. (2021). Racial and ethnic differences in COVID19 vaccine hesitancy and uptake (p. 2021.02.25.21252402). https://doi.org/10.1101/2021.02.25.21252402

Niño, M. D., Hearne, B. N., \& Cai, T. (2021). Trajectories of COVID19 vaccine intentions among U.S. adults: The role of race and ethnicity. SSM - Population Health, 15, 100824. https://doi.org/ 10.1016/j.ssmph.2021.100824

Quinn, S. C., Jamison, A. M., An, J., Hancock, G. R., \& Freimuth, V. S. (2019). Measuring vaccine hesitancy, confidence, trust and flu vaccine uptake: Results of a national survey of White and African American adults. Vaccine, 37, 1168-1173. https://doi.org/10. 1016/j.vaccine.2019.01.033

Quinn, S. C., Jamison, A., Freimuth, V. S., An, J., Hancock, G. R., \& Musa, D. (2017). Exploring racial influences on flu vaccine attitudes and behavior: Results of a national survey of White and African American adults. Vaccine, 35, 1167-1174. https://doi.org/ 10.1016/j.vaccine.2016.12.046

Savoia, E., Piltch-Loeb, R., Goldberg, B., Miller-Idriss, C., Hughes, B., Montrond, Am., Kayyem, Jn., \& Testa, Ma. (2021). Predictors of COVID-19 vaccine hesitancy: Socio-demographics, co-morbidity and past experience of racial discrimination [Preprint]. Public and Global Health. https://doi.org/10.1101/2021.01.12.21249152

Smith, T. W., Davern, M., Freese, J., \& Morgan, S. L. (2019). General social surveys, 1972-2018: Cumulative codebook. NORC.

Ta Park, V. M., Dougan, M., Meyer, O. L., Nam, B., Tzuang, M., Park, L. G., Vuong, Q., \& Tsoh, J. Y. (2021). Vaccine willingness: Findings from the COVID-19 effects on the mental and physical health of Asian Americans \& Pacific Islanders survey study (COMPASS). Preventive Medicine Reports, 23, 101480. https:// doi.org/10.1016/j.pmedr.2021.101480

Walker, K. K., Owens, H., \& Zimet, G. (2020). We fear the unknown: Emergence route and transfer of hesitancy and misinformation among HPV vaccine accepting mothers. Preventive Medicine Reports, 20, 101240. https://doi.org/10.1016/j.pmedr.2020. 101240

Wallace, L. S., Rogers, E. S., Roskos, S. E., Holiday, D. B., \& Weiss, B. D. (2006). Brief report: Screening items to identify patients with limited health literacy skills. Journal of General Internal Medicine, 21, 874-877. https://doi.org/10.1111/j.1525-1497.2006. 00532.x
Warren, R. C., Forrow, L., Hodge, D. A., \& Truog, R. D. (2020). Trustworthiness before trust-Covid-19 vaccine trials and the black community. New England Journal of Medicine, 383, e121. https:// doi.org/10.1056/NEJMp2030033

Washington, H. A. (2006). Medical apartheid: The dark history of medical experimentation on black americans from colonial times to the present. Doubleday.

Washington, J. (2020). New poll shows Black Americans put far less trust in doctors and hospitals than white people. The Undefeated. https://theundefeated.com/features/new-pollshows-black-americans-put-far-less-trust-in-doctors-and-hospi tals-than-white-people/

WHO. (2019a). Ten threats to global health in 2019. World Health Organization. https://www.who.int/vietnam/news/feature-stories/ detail/ten-threats-to-global-health-in-2019

WHO. (2019b). Measuring Behavioural and Social Drivers (BeSD) of Vaccination Working Group (pp. 1-8) [Meeting Report]. World Health Organization. https://www.who.int/immunization/progr ammes_systems/Meeting_report_May2019.pdf

WHO EURO. (2011). Report of the SAGE Working Group on Vaccine Hesitancy. Working Group on Vaccine Communications. https://www.who.int/immunization/sage/meetings/2014/octob er/1_Report_WORKING_GROUP_vaccine_hesitancy_final.pdf

Williams, R. (2006). Generalized ordered logit/partial proportional odds models for ordinal dependent variables. The Stata Journal: Promoting Communications on Statistics and Stata, 6, 58-82. https://doi.org/10.1177/1536867X0600600104

Willis, D. E., Andersen, J. A., Bryant-Moore, K., Selig, J. P., Long, C. R., Felix, H. C., Curran, G. M., \& McElfish, P. A. (2021). COVID19 vaccine hesitancy: Race/ethnicity, trust, and fear. Clinical and Translational Science, 00(00), 1-8. https://doi.org/10.1111/cts. 13077

Ylitalo, K. R., Meyer, M. R. U., Lanning, B. A., During, C., Laschober, R., \& Griggs, J. O. (2018). Simple screening tools to identify limited health literacy in a low-income patient population. Medicine, 97, e0110. https://doi.org/10.1097/MD.0000000000010110

Publisher's Note Springer Nature remains neutral with regard to jurisdictional claims in published maps and institutional affiliations. 